prescribed a controlled drug, and therefore by implication a drug of addiction, is also likely to cause many to stop taking their phenobarbitone. All these factors would expose the patien to the risk of status epilepticus. Even the slow withdrawal of the drug does not in our experience protect patients from this eventuality, which still carries a high mortality and morbidity.

Phenobarbitone is not a drug that is abused and, because of its pharmacokinetic properties, it is unlikely to be. Its inclusion in the proposed legislation would lead to the anomalous situation whereby the prescribing of this compound would be controlled, and yet that of the prodrug primidone (Mysoline) would be unaffected. Abuse of primidone has been reported in the USA.

It has been argued that inclusion of phenobarbitone in the legislation would facilitate analytical work by the forensic laboratories, as a simple screening test would suffice rather than specific identification of individual compounds. In fact, it is relatively easy to separate out the various barbiturates by a variety of chromatographic techniques. But we feel particularly that the blanket inclusion of phenobarbitone purely for laboratory convenience is not a satisfactory reason for legislating against a drug of non-abuse. This action would cause considerable embarrassment and inconvenience to people with epilepsy if they were found in possession of a controlled drug which, in fact, was being prescribed for sound medical reasons. The discrimination against people with epilepsy is extensive enough without adding to it in this way.

Although as ex-CURB campaigners we fully support the attempts to restrict the abuse of the hypnotic barbiturates, we do not feel that this should be achieved at the expense of a large number of people handicapped by epilepsy.

The Thorn Epilepsy Research Group, Institute of Neurology,

Queen Square, $3 B G$

AlAN Richens

Chalfont Centre for Epilepsy,

Chalfont St Peter,

***The Secretary writes: "In the publicity the BMA has given to the inclusion of barbiturates in the Misuse of Drugs Act it has emphasised that phenobarbitone remains one treatment of choice for epilepsy. Surely it is not unreasonable to ask doctors to write phenobarbitone prescriptions for those of their patients who need them and withdrawal of the drug because of this change seems unlikely. The BMA sounded the views of all branches of the profession before deciding on this policy, though it could well be that no representative of the Medical Advisory Committee of the British Epilepsy Association was personally approached, an omission the BMA regrets."-ED, $B M F$.

\section{Epileptic drugs and the police}

SIR,-May we add our voice to that of $\mathrm{Dr}$ J A Riddle (12 January, $p$ 113) in appealing to doctors to supply their patients with a statement if they are in possession of a driving licence?

We could add to the case he quotes several others concerning people (usually young people and often male) who have been kept overnight in police cells without medication after having been found in some sort of epileptic seizure. These people also could benefit from a medical statement. We agree with your correspondent's statement that an official form would be preferable, but in view of the length of time it takes to produce such a form it would be extremely helpful if doctors could fill the gap. This association has in fact written to the Driver and Vehicle Licensing Centre in Swansea to ask for co-operation in this matter.

OWEN M JONES

British Epilepsy Association,

\section{Diabetic impotence}

SIR,-We are extremely interested in the results reported by $\mathrm{Dr} \mathrm{D} J$ Hosking and others ( 1 December, p 1394) but concerned about their conclusion.

On the basis of an overlap between their diabetic and normal groups, they conclude that impotence in most diabetics is likely to be psychological rather than organic. This appears to be based on the following assumptions: (1) The occurrence of nocturnal erections distinguishes between organic and psychogenic cases. While an interesting possibility this remains to be demonstrated. The assumption that those cases in which normal sleep erections occur are psychogenic has introduced a circularity into much of this reasoning. (2) All types of organic pathology would produce the same effects on nocturna erections. (3) Nocturnal erections during sleep in normal men are full erections and sufficien for intercourse.

None of these assumptions can be justified with the evidence at present available. While these results suggest that complete impairmen of erections is not necessarily associated with diabetic impotence, the effects of either autonomic neuropathy, peripheral vascular disease, or some other pathology could interfere with the attainment of full (that is, stiff) erections and could consequently lead to anxiety about performance, which in its tur produces further impairment. There is still much to be learnt before we understand erectile failure, diabetic or otherwise.

JOHN BANCROFT

MRC Reproductive Biology Unit,

Centre for Reproductive Biology,
Edinburgh EH3 9EW

F C W WU

Royal Infirmary,
Edinburgh EH3 $9 \mathrm{YW}$

\section{Intermittent venous sampling during} exercise

SIR,-Drs M J G Farthing and Anna M Mattei (3 November, p 1112) and Drs D P Mikhailidis and R A Hutton (12 January, p 111) have reported experience using different techniques for intermittent venous sampling in hospital patients. When samples are required from subjects undertaking active exercise there is the additional problem of "the moving target." Using butterfly needles (gauge 19-21, tubing length $305 \mathrm{~mm}$ ) (Abbott Laboratories) we have been able to obtain multiple samples over periods of up to two hours from subjects exercising on a cycle ergometer. ${ }^{1}$ The butterfly is carefully positioned in a superficial vein of the forearm away from a joint. The tubing is occluded with a compression clip. After sampling the system is flushed with sterile $0.9 \%$ saline.

The flexible narrow-diameter tubing attached to the butterfly needle is of sufficient length to allow the subject reasonable freedom of movement at the time of sampling, and also provides a system of small volume which may be flushed easily. We have not encountered troublesome clot formation in the lumen of any catheter well flushed with saline after blood sampling. This simple inexpensive technique has been used successfully in more than 30 subjects exercising at mild, moderate, or severe work intensities.

R J RATCLIFFE P L T WILLAN

University Department of Anatomy,

Manchesster M13 9PT

Ratcliffe

\section{Eating and ulcers}

SIR,-It is true, as you say in your leading article (26 January, p 205), that much dietary advice to patients with peptic ulceration "is more often based on impression and prejudice than on fact." Moreover, what little sound evidence does exist suggests that special diets are ineffective. ${ }^{1}$ But this may well be because the wrong sorts of diet have been used.

Experience with the use of a low-carbohydrate diet for the treatment of obesity ${ }^{2}$ led to the unexpected observation that any concurrent dyspepsia, associated, for example, with peptic ulceration or hiatus hernia, was in many patients relieved as soon as the diet was adopted. As a result, we instituted a controlled trial in which alternate chronically dyspeptic patients with or without demonstrable ulceration were instructed to follow either the standard hospital "gastric diet" or a diet in which carbohydrate was restricted to $60 \mathrm{~g}$ or $70 \mathrm{~g}$ a day. At the end of three months the diets were reversed.

As we have reported in a brief communication $^{3}$ to the Nutrition Society, of the 41 patients who completed the trial, two reported that their symptoms were improved more with gastric diet, 11 found no difference, and 28 found that they were better with the lowcarbohydrate diet. The chief difference between the diets was the high proportion of starch and sugar (sucrose) in the gastric diet and the low proportion of these constituents in the low-carbohydrate diet. In order to see whether it was the reduction of dietary starch or the reduction of dietary sugar that was the more likely cause of the symptomatic improvement with the low-carbohydrate diet, we examined the gastric juice of seven healthy young men who were volunteers in an experiment in which their dietary sugar was increased and their dietary starch decreased by a similar amount. ${ }^{4} \mathrm{~A}$ test meal $^{5}$ carried out before breakfast revealed that, after two weeks on the high-sugar diet, there was an increase in peak gastric acidity by about $20 \%$ and an increase in peak pepsin activity by about $250 \%$.

Thus we can now say that, while it is true that conventional dietary treatment is ineffective in relieving the symptoms of chronic dyspepsia, it is not true that all dietary treatment is ineffective. But it remains to be seen whether a diet that is low in carbohydrate, and 